\title{
The power of vivid experience in hand hygiene compliance
}

Authors:

Paul W. Nicol

Rochelle E.Watkins

Robert J. Donovan

Dianne Wynaden

Helen Cadwallader

Division of Health Sciences, Curtin University of Technology

Corresponding author: Paul W. Nicol, 42 Kersey Way, Carine, WA 6020 e-mail pnicol@iinet.net.au

Running title: Power of vivid experience 


\section{The power of vivid experience in hand hygiene compliance}

\section{Summary}

In recent years, explicit behavioural theories have been used in some research into hand hygiene behaviour. One of the most prominent of these has been the Theory of Planned Behaviour (TPB). In this qualitative study aimed at increasing understanding of infection control practices in the acute care setting, the TPB was identified as a suitable framework for the emergence of new insights that have the potential to improve the power of existing education and training.

The theory emerging from the research was based on a finding that individual experience is of greater import than formal education in explaining hand hygiene behaviour. This indicated that exposure to vivid vicarious experience is a potential means to improving the power of existing training methods and increasing the propensity for instilling sustainable adequate hand hygiene habits.

Key words: hand hygiene, Theory of Planned Behaviour, infection control

\section{Introduction}

Of the many measures to prevent the spread of antimicrobial-resistant pathogens in hospitals and reduce hospital-acquired infections, hand hygiene is considered to be

primary. ${ }^{1-3}$ The challenge of ensuring compliance with the requirements of adequate hand cleansing is well-documented and a considerable body of research into the hand hygiene behaviour of health care workers in the hospital setting has been established. However, although a growing number of these studies indicate success to varying extents, methods for sustained improvement remain elusive ${ }^{4}$ and the studies include few in-depth explorations of the factors that influence infection control behaviour. It appears that there is limited understanding of the motivating factors that influence infection control behaviour. ${ }^{5}$

These motivating factors are complex, which is why the most successful interventions to improve compliance have been multi-faceted. ${ }^{1}$ An explicit theoretical research framework can assist in making sense of the multiplicity of factors that can influence hand hygiene behaviour, but this approach has been comparatively infrequent. ${ }^{6}$ Those 
studies that have been grounded in theory have tended to be based on social cognition models, primarily the Theory of Planned Behaviour (TPB). ${ }^{7-11}$

\section{Method}

The objective of the research was to increase understanding of health care professionals' (HCP) infection control practices in the acute care setting. Grounded theory was used to explore the motivational bases of HCPs' behaviour and enable indepth exploration of multiple subjective experiences. ${ }^{12}$ The grounded theory method enables complexity of behaviour to be captured without the restraints of preconceived theories or models. ${ }^{13-16}$ Concepts emerge as the researcher develops his or her perspective ${ }^{15}$ and the emphasis is on generation of substantive theory through constant comparison data analysis and interpretation of relationships between identified categories or groups. ${ }^{17,18}$

\section{Setting and sample}

This study was conducted in two general medical and surgical wards in each of two metropolitan teaching hospitals between December 2006 and December 2007. Purposive sampling methods ${ }^{19}$ were used to recruit 33 nurses (31 female, 2 male) and 11 doctors (7 male, 4 female), and a physiotherapist and a phlebotomist (both female). As the research progressed, theoretical sampling was used to collect more refined observation data and relevant documentation as determined by the iterative process of constant comparison, until saturation ${ }^{19}$ was reached, where no new information relevant to the purpose of the study was gained from additional sampling.

\section{Data collection}

Semi-structured interviews were audio recorded. Interview content was principally determined by the interview participants (IPs) within the framework of an interview guide. We also used observational data and documentation from nearly 60 hours of site visits over a three-month period in to complement and inform the interviews ${ }^{20}$ with contextual information, including staff interactions and social, environmental and organisational influences.

\section{Ethics}


Clinical staff were informed about the study and participants recruited using staff noticeboards and face-to-face communication. Permission to conduct observations was obtained from hospital administration and clinical staff in charge of each area. IPs consented to audio recording of interviews. Informed consents complied with hospital and university ethics protocols.

\section{Analysis}

Interview transcripts and observational notes were analysed employing the NVivo qualitative analysis software package. The first transcripts were scanned for emergent concepts, which informed subsequent interviews, observations and analysis. Once saturation was reached and identified categories were expansive and complete, substantive theory was developed. Relevant literature was then searched to see whether there were extant theoretical models in which the emergent concepts and substantive theory could be situated in order to integrate the theory with existing knowledge.

In the findings, the emergent concepts and themes are printed in italics.

\section{Findings}

The infection control measure that IPs identified to be of primary importance in everyday practice was hand hygiene, including the use of gloves. Despite this all considered that their own hand hygiene practice, while above average, was less than optimal.

\section{Competence}

Knowledge of general hand hygiene principles appeared high, but knowledge of what to do in specific circumstances and when to do it, termed competence in this study, appeared variable and was a limiting factor for some IPs' practice. IPs generally considered that a basic pre-requisite for competence, accessibility to consumables and equipment, was satisfactory in the hospitals, where sinks with soap-based cleansing liquids as well as alcohol hand cleansing solution (AHCS) bottles and three-size glove dispensers were available inside, and/or outside the doors, of patient rooms. 
Both interview and observational data revealed that some participants had appropriate hand hygiene habits deeply instilled. For these people, failure to perform adequate hand hygiene tended to manifest as a feeling that something was "not right", a feeling which sometimes preceded a conscious realisation of the deficiency. It was evident from both observations and self-report that routine hand-cleansing behaviour, whether adequate or not, was often non-conscious, the IP being unaware of taking the action. However, they would adapt their practice to non-routine circumstances and this tended to be perceived as a conscious decision that IPs called "common sense”.

\section{Concurrence}

Knowledge of why hand hygiene measures were necessary and a commitment to them was termed concurrence. The level of concurrence was influenced directly by formal training and education, which had had a profound effect on attitudes of some of the IPs. IPs believed that continual repetition in training was necessary both in forming and maintaining appropriate habits.

However, the aspects of training that IPs most recalled were experiential, such as visual demonstrations of hand-washing ineffectiveness. They perceived that, in the absence of sufficiently compelling evidence to which they could relate, many HCPs would fail to adequately associate performance of hand hygiene practices with transmission of infection. Several IPs indicated that regular dissemination of convincing evidence about the effectiveness of hand hygiene would increase their motivation to improve hand hygiene performance. Evidence could take the form of narratives about particular incidents or statistics on performance.

Closely related to this concept was the influence of the immediacy of outcomes and/or their visibility/tangibility. For example, some IPs reported that they were more stringent with the handling of sharps than with hand hygiene because the outcome of a mishap with sharps was immediately apparent.

However, the most powerful influence appeared to be direct vivid experience, such as a personal exposure to an outbreak of infection in the hospital or in a patient under the IP's care. IPs reported that the emotional impact of this permanently heightened their awareness and resulted in a sustained improvement in hand hygiene practice. 
Experience of working with patients who were perceived as particularly vulnerable, such as in an oncology, haematology or paediatric ward, had a similar effect.

Such experiences influenced a further important motivating factor, recognition of the need to protect oneself and/or others. Usually, protection of oneself and family were considered more important than the protection of others, although the close relationship between patient protection and self-protection was recognised.

Social influences, especially from childhood, were another major influence on both practice of infection control and acceptance of training. For people whose upbringing had included strict hand washing habits, training in hand hygiene reinforced their existing tendencies.

\section{Other attitudinal influences}

Desensitisation to risk as a consequence of long-term familiarity with potential infection transmission situations without apparent adverse consequences affected IPs' attitude to infection transmission and hand hygiene practice. A variation of this was a belief that one was unlikely to be personally infected, which IPs dubbed the "Superman theory.” Other factors were a personal sense of responsibility, particularly work ethic and morality, and emotional involvement, presented as compassion and/or a need to feel useful.

\section{Organisational culture}

Role models, particularly senior staff, peer pressure and, for nurses, the influence of preceptors and buddies had a major effect on beliefs about hand hygiene. It was perceived that differences in the interpretation of hand hygiene guidelines within and between wards contributed to a lack of standardisation in what role models considered to be appropriate practice.

Of further influence were beliefs about the ease or difficulty of practice. This was principally reflected in a belief that a lack of time, due to what was termed "busyness”, precluded adequate hand hygiene standards. While grounded in actual difficulties experienced in wards, this had become an unwritten assumption among 
HCPs and tended to be used as a catch-all justification - or excuse - for inadequate practice.

Most IPs considered that organisational reinforcement, the hospitals' support of good practice, was positive, although this appeared to have had little effect on attitudes and predispositions.

\section{Situational influences}

IPs perceived a number of factors in the workplace situation that had an ongoing effect on their practice. Facilitating factors included situational cues to action, which are signals in the working environment that prompt a response, e.g., highly visible AHCS bottles, visible soiling and smells, signs and posters. Inhibitors included events or risk conducive circumstances that act to prevent an intended hand hygiene action from occurring, such as focusing on an immediate problem or on one aspect of treatment or care to exclusion of others, competition for attention and prioritising, inaccessibility of consumables and equipment, interruptions, inappropriate routines and fatigue.

\section{Theoretical model}

The TPB model emerged as the most suitable framework to aid understanding of the relationships between multiplicity of influences and motivational factors that emerged from our analysis and to present the substantive theory. In the TPB, performance depends upon the strength of an individual's intention, or predisposition, to perform an action. These predispositions are influenced primarily by 1) personal perceptions or beliefs about the advantages and disadvantages of the behaviour and its consequences, and 2) subjective norms, which are beliefs about what others would expect the individual to do in the situation. A third influence is perceived control, or self-efficacy, i.e., beliefs about the ease or difficulty of performance.

In our study, beliefs grounded in concurrence, vivid experience, desensitisation, a personal sense of responsibility and emotional involvement were the basis of IPs' attitudes to the risks associated with poor hand hygiene and their responsiveness to them. Beliefs influenced by role models and a shared assumption about the ease or difficulty of performance were both influenced by, and an outcome of, organisational 
cultural (social) norms. Beliefs about whether the IP could perform hand hygiene appropriately (self-efficacy) were mainly influenced by his or her perception of personal competence (Figure 1)

However, it was apparent from the self-reported discrepancy between intention and behaviour that our analysis also needed to explore the factors influencing the translation of intention into actual behaviour. Several studies have provided evidence that, while supporting its predictive power with regard to hand hygiene intention, the

TPB is less effective in explaining the translation of intention into practice. 567212223 A comprehensive model based on the TPB would therefore have to try to account for these discrepancies between predisposition and performance. Our findings show that IPs identified several situational influences that profoundly and continually affected the transition between intended and actual behaviour. These situational factors are shown as additional to the standard TPB model (Figure 1).

In the model, boxes to the left represent the major beliefs that inform the three TPB factors influencing intention. Each of these constructs encompasses several subcomponents which for our hospitals identify and describe in detail specific beliefs. This rich data source can be used to inform interventions at a specific level. For example, our findings showed that a lack of standardisation of beliefs among senior nursing staff perceived to be role models was leading to different interpretations of infection control guidelines and some confusion about correct practice among some junior staff.

$<$ Insert Figure 1 here $>$

\section{Discussion}

The use of the TPB model as a framework reveals a wide range of perspectives and difference in practice, which reinforces the contention that interventions to improve hand hygiene practice should be multi-faceted ${ }^{1}$, in order to influence the maximum number of HCPs. The model integrates factors which have previously been documented with some which have received minimal attention, including the automaticity of much hand hygiene behaviour. 
We observed that much hand hygiene education appears to be based on an assumption that actions occur mainly after conscious deliberation. In social psychology, the theory that conscious intention or reasoned decision-making is the primary factor determining behaviour has been increasingly questioned in the last two decades and a considerable body of research indicates that much behaviour occurs automatically, often as a response to environmental cues. ${ }^{25-27}$ The TPB is consistent with these theories of behavioural automaticity, ${ }^{24}$ with behaviour being influenced by attitude activation. Automaticity is evident in habitual behaviour as well as in unmediated or non-conscious responses to influences from the environment. ${ }^{27,28}$ This has important ramifications for instilling sustainable appropriate hand hygiene habits, because predispositions, or intentions, need to be strong enough to consistently ensure that adequate hand hygiene practice is a habitual or reflex response.

Additionally, context is an influence: steady and frequent repetition of behaviour is not a necessary indication that a habit would be followed if the situation or context changes. ${ }^{29}$ Conscious intentions or reasoning may override the non-conscious in situations where, e.g., habits and intentions conflict or when perceptions of ease outweigh the effort to produce a more valuable outcome. This was evident in the circumstance-based application of "common sense" by the IPs.

In our modification of the TPB framework, behavioural change can be affected by interventions that affect the both the situational environment and the strength of intention. Our findings indicated that there were ongoing interventions in the hospitals' situational environments, e.g., re-positioning of AHCS bottles and development of visual reminder cues. However, a major factor that could influence predisposition appeared to have received less attention. In our study, individual experience, particularly vivid episodes, was perceived to have been a persistent positive influence in instilling sustained improvements in hand hygiene practice by strengthening attitudes and intentions, and was a more powerful driver of hand hygiene behaviour than current formal training. Our data suggests that the introduction of forms of vivid experience to the learning process would, by 
strengthening predisposition, improve responses to cues to action and lessen the impact of inhibitors.

Individual experiential learning, in contrast to learning by assimilation, has long been recognised as necessary to promote organisational change. ${ }^{30}$ However, assimilative rather than experiential hand hygiene education and training appears to be the current norm. IPs opined that habits needed to be embedded during training, but the education they received had been inconsistently effective in achieving this.

The deep-seated nature of habits was recognised by IPs who acknowledged the importance of childhood experiences in the formation of their predispositions both to hand hygiene practice and to their receptiveness to training. This inherent hand hygiene behaviour ${ }^{11,31}$ could become so habitual that it would be unusual for an individual to consciously develop an intention to wash hands. Rather, it was finding the means to perform the action that sometimes required conscious effort. Elective hand hygiene, on the other hand, is counter-intuitive, because it is performed when there is no intuitive need for it, ${ }^{11,31}$ and requires training to be activated. From the IPs' self-reports, learning from experience was an effective means of transforming that elective behaviour into inherent.

It is our contention that the introduction of experiential elements would improve the effectiveness of existing training programs. The impact of direct exposure to actual adverse outcomes could be incorporated through the use of vicarious experience via exposure to graphic, emotion-arousing narratives and/or videos of events or the results of inadequate infection control.. ${ }^{6}$ The use of graphic images to elicit the vicarious experience of the emotions and suffering of persons with life-threatening illnesses has been used with considerable success in advertising and other communication channels in a number of public health areas, most notably in anti-smoking and road safety campaigns. ${ }^{32}$ Similar exposure in hand hygiene education could usefully be reinforced by meeting IPs’ expressed need for compelling evidence of the morbidity, mortality and costs associated with hospital-acquired infections. 
Our study was conducted in Western Australian hospitals, which have relatively low rates of hospital-acquired epidemic MRSA. ${ }^{33} 34$ Quite different perceptions may be obtained in other situations. Nevertheless, the overall TPB framework is transferable.

\section{Conclusion}

Exposure to vivid vicarious experience is a potential means to improving the power of existing training methods and significantly increasing the propensity for instilling sustainable adequate hand hygiene habits. These are factors that have received little attention elsewhere.

\section{Acknowledgements}

This paper is dedicated to the memory of our colleague and friend, Professor Aileen Joy Plant, who was chief investigator for this research until her sudden death on 27 March 2007 while on a mission for the World Health Organization. Her outstanding vision for and contribution to the control of infectious disease is greatly missed.

We thank all the participants and the people at Fremantle and Sir Charles Gairdner Hospitals whose willing assistance helped make the project possible.

The project has been funded by the National Health and Medical Research Council of Council of Australia. 


\section{References}

1. Pittet D, Allegranzi B, Sax H, et al. Evidence-based model for hand transmission during patient

care and the role of improved practices. The Lancet Infectious Diseases 2006;6(10):641-652.

2. Sax H, Allegranzi B, Uckay I, Larson E, Boyce J, Pittet D. 'My five moments for hand hygiene': a user-centred design approach to understand, train, monitor and report hand hygiene. J Hosp Infect 2007;67(1):9-21.

3. Gould DJ, Hewitt-Taylor J, Drey NS, Gammon J, Chudleigh J, Weinberg JR. The CleanYourHandsCampaign: critiquing policy and evidence base. J Hosp Infect 2007;65(2):95-101.

4. Whitby M, McLaws M-L, Slater K, Tong E, Johnson B. Three successful interventions in health care workers that improve compliance with hand hygiene: Is sustained replication possible? Am J Infect Control 2008;36(5):349-355.

5. Berhe M, Edmond MB, Bearman GML. Practices and an assessment of health care workers' perceptions of compliance with infection control knowledge of nosocomial infections. Am J Infect Control 2005;33(1):55-57.

6. Pittet D. The Lowbury lecture: Behaviour in infection control. J Hosp Infect 2004;58:1-13.

7. O'Boyle CA, Henly SJ, Larson E. Understanding adherence to hand hygiene recommendations: The Theory of Planned Behavior. Am J Infect Control 2001;29(6):352-360.

8. Pittet D, Simon A, Hugonnet S, Pessoa-Silva CL, al e. Hand hygiene among physicians: Performance, beliefs, and perceptions. Ann Intern Med 2004;141(1):1-8. 9. Pessoa-Silva CL, Posfay-Barbe K, Touveneau S, Perneger TV, Pittet D. Attitudes and perceptions toward hand hygiene among healthcare workers caring for critically ill neonates. Infection Control \& Hospital Epidemiology 2005;26(3):305311.

10. Sax H, Uckay I, Richet H, Allegranzi B, Pittet D. Determinants of good adherence to hand hygiene among healthcare workers who have extensive exposure to hand hygiene campaigns. Infect Control Hosp Epidemiol 2007;28(11):1267-1274.

11. Whitby M, Pessoa-Silva CL, McLaws M-L, et al. Behavioural considerations for hand hygiene practices: the basic building blocks. J Hosp Infect 2007;65(1):1-8.

12. Whiteley A, McCabe M, Buoy L, et al. Planning the qualitative research interview. Curtin University of Technology Graduate School of Business Working Paper Series 98011998.

13. Becker PH. Pearls, pith and provocation: common pitfalls in grounded theory research. Qualitative Health Research 1993;3(2):254-260.

14. Glaser BG. Basics of grounded theory analysis. Mill Valley, California:

Sociology Press; 1992.

15. Robrecht LJ. Grounded theory: evolving methods. Qualitative Health Research 1995;5(2):169-177.

16. Whiteley A. Grounded research: a modified grounded theory for the business setting. Curtin University of Technology Graduate School of Business Working Paper Series 00022000.

17. Glaser BG. The grounded theory perspective: conceptualization contrasted with description. Mill Valley, California: Sociology Press; 2001. 
18. Strauss A, Corbin J. Basics of qualitative research: grounded theory procedures and techniques. Newbury Park, California: Sage Publications; 1990.

19. Glaser B. Doing grounded theory: Issues and discussions. Newbury Park, California: Sociology Press; 1998.

20. Streubert H, Carpenter D. Qualitative research in nursing: advancing the humanistic imperative. 2nd ed. Philadelphia: Lippincott; 1999.

21. Sheeran P, Conner M, Norman P. Can the Theory of Planned Behavior explain patterns of health behavior change? Health Psychol 2001;20(1):12-19.

22. Kretzer EK, Larson EL. Behavioral interventions to improve infection control practices. Am J Infect Control 1998;26(3):245-253.

23. Jenner EA, Watson PWB, Miller L, Jones F, Scott GM. Explaining hand hygiene practice: an extended application of the Theory of Planned Behaviour. Psychology, Health \& Medicine 2002;7(3):311-326.

24. Ajzen I, Fishbein M. Attitudes and the attitude-behavior relation: Reasoned and automatic processes. European Review of Social Psychology 2000;11(1):1-33.

25. Aarts H, Dijksterhuis A. Habits as knowledge structures: Automaticity in goal-directed behavior. J Pers Soc Psychol 2000;78(1):53-63.

26. Bargh JA, Chartrand TL. The unbearable automaticity of being. Am Psychol 1999;54(7):462-479.

27. Bargh JA, Ferguson MJ. Beyond behaviorism: On the automaticity of higher mental processes. Psychol Bull 2000;126(6):925-945.

28. Fazio RH, Sanbonmatsu DM, Powell M, Kardes FR. On the automatic activation of attitudes. J Pers Soc Psychol 1986;50(2):229-238.

29. Ouellette JA, Wood W. Habit and intention in everyday life: The multiple processes by which past behaviour predicts future behaviour. Psychol Bull 1998;124(1):54-74.

30. De Geus AP. The living company. Boston, Mass.: Longview Publishing; 1997.

31. Whitby MM, McLaws M-LM, W. Ross MM. Why healthcare workers don't wash their hands: A behavioral explanation. Infect Control Hosp Epidemiol 2006;27(5):484-492.

32. Dunlop SM, Wakefield M, Kashima Y. The contribution of antismoking advertising to quitting: Intra- and interpersonal processes. Journal of Health Communication 2008;13(3):250-266.

33. Jarvis WR. The United States approach to strategies in the battle against healthcare-associated infections, 2006: transitioning from benchmarking to zero tolerance and clinician accountability. J Hosp Infect 2007;65(Supplement 2):3-9. 34. Boyce JM, Cookson B, Christiansen K, et al. Meticillin-resistant Staphylococcus aureus. The Lancet Infectious Diseases 2005;5(10):653-663. 
Figure 1

Theory of Planned Behaviour model for hand hygiene practice

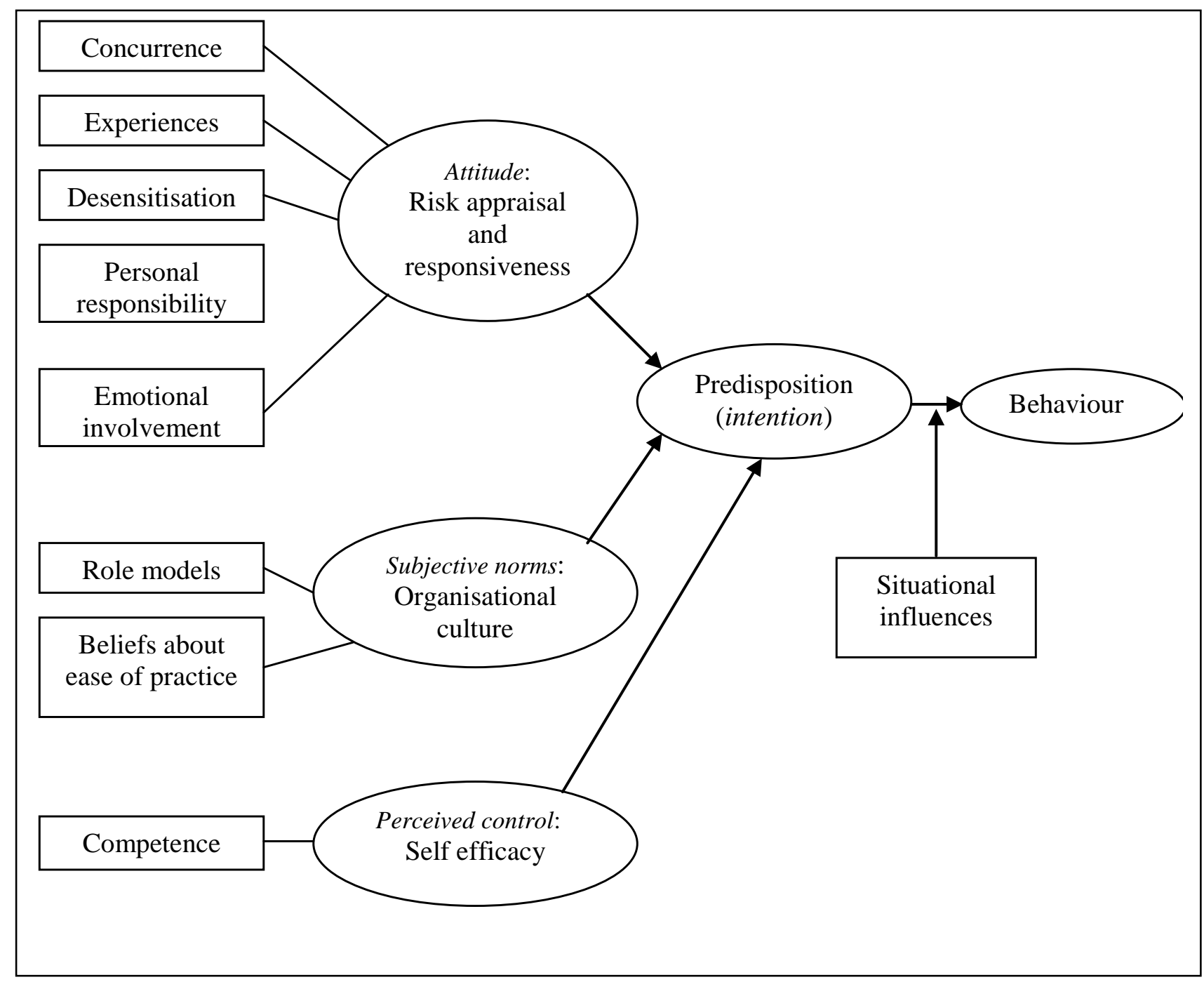

